Eixo Temático: Biologia Aplicada

\title{
ET-09-022 \\ RELAÇÃO PESO-COMPRIMENTO DE 59 ESPÉCIES DE PEIXES DO ESTUÁRIO DO RIO MAMANGUAPE, PARAÍBA, BRASIL
}

Vivianne Evelyn do Nascimento Alves*, Ana Luísa Araújo de Amorim, Ana Lúcia Vendel

Centro de Ciências Biológicas e Sociais Aplicadas. Universidade Estadual da Paraíba. Campus V. Rua Horácio Trajano de Oliveira, S/N. Cristo Redentor. João Pessoa-PB. *E-mail: evelyn_vivianne@hotmail.com.

http://dx.doi.org/10.21472/congrebio2016.et-09-022

\section{RESUMO}

Foram obtidas as relações peso-comprimento de 59 espécies de peixes teleósteos no Estuário do Rio Mamanguape. Um total de 3.638 indivíduos pertencentes a 25 famílias foram medidos e pesados. As famílias mais representativas foram Engraulidae e Gobiidae, com 7 espécies cada, seguidas por Gerreidae, com 5 espécies. Significativas relações pesocomprimento com altos coeficientes de correlação foram encontrados para a maioria das espécies tanto na estação seca $\left(r^{2}=0,9684\right)$, quanto na chuva $\left(r^{2}=0,9997\right)$. Este estudo relata as primeiras conclusões sobre a relação peso-comprimento da comunidade de peixes neste estuário.

Palavras-chave: Engraulidae; Gobiidae; Gerreidae; APA de Mamanguape.

\section{INTRODUÇÃO}

Os estuários representam áreas vitais de alimentação, reprodução e crescimento para várias espécies de peixes, proporcionando ainda locais de refúgio para os estágios mais vulneráveis a predação (GODEFROID et al., 2003). Desta forma, abrigam principalmente organismos juvenis, pois são locais onde os mesmos obtêm proteção e alimento, elevando assim as suas chances de sobrevivência (KENNISH, 1990).

A ictiofauna estuarina desempenha um importante papel ecológico, através da condução de energia de níveis tróficos inferiores para os superiores, troca de energia com os ecossistemas vizinhos ou armazenamento de energia através das espécies que passam parte da sua vida nesses ambientes (ARAÚJO et al., 2004). Ela é normalmente caracterizada por espécies residentes, migrantes marinhas e de água doce que utilizam o estuário como áreas de alimentação, reprodução e criação de larvas (BLABER, 2000).

A relação peso-comprimento de uma comunidade pode fornecer informações básicas sobre a biomassa de peixes e os estoques pesqueiros (VIANNA et al., 2004), sendo de grande importância, pois fornece informações necessárias para compreender as relações ecológicas das espécies (CORRÊA et al., 2016), servindo como ferramenta para os estudos da biologia pesqueira, necessária para o manejo e gestão dos ambientes estudados (LIZAMA e AMBRÓSIO, 2003).

O objetivo deste estudo foi fornecer, pela primeira vez, dados de referência sobre a relação peso-comprimento para várias espécies de teleósteos estuarinos juvenis ocorrentes no estuário do rio Mamanguape, dentre os quais há muitas espécies que são alvos da pesca artesanal e comercial na região. 


\section{METODOLOGIA}

O estudo foi desenvolvido em um sistema de transição, localizado no Nordeste do Brasil - Estuário do Rio Mamanguape (Figura 1). O clima da região é tipo AS’ de acordo com Köppen, ou seja, quente e úmido. A precipitação varia entre $2000 \mathrm{~mm} /$ estação para menos de 30 $\mathrm{mm} /$ estação. A estação chuvosa começa em fevereiro e termina em julho, com maior precipitação de abril a junho, enquanto a estação seca é de agosto a janeiro, com precipitação mínima de outubro a dezembro. A média anual da temperatura da água é entre $24{ }^{\circ} \mathrm{C}$ e $26^{\circ} \mathrm{C}$.

a. SECA

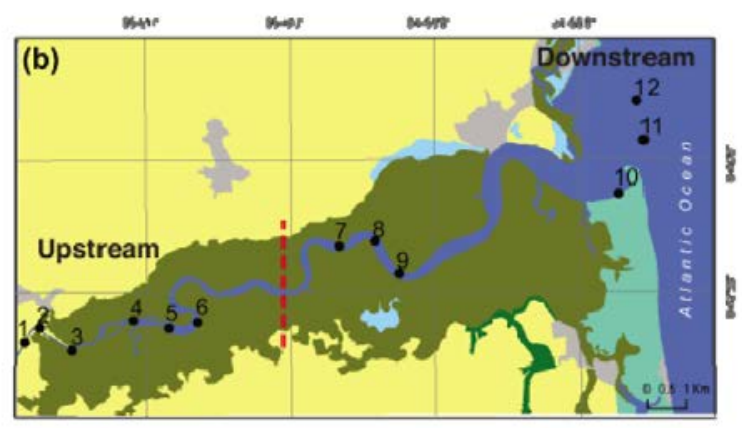

b. CHUVA

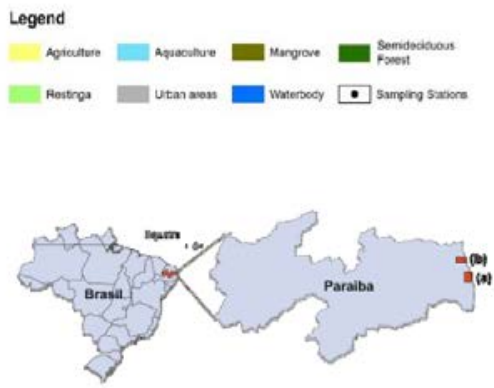

Figura 1. Estuário do Rio Mamanguape, PB.

O Estuário do Rio Mamanguape é considerado o segundo maior do Estado da Paraíba e está localizado dentro da Área de Proteção Ambiental (APA) da Barra do Rio Mamanguape, cujo principal objetivo é proteger os habitats costeiros e o peixe-boi marinho Trichechus manatus Linnaeus, 1758. Na borda da área de mangue, existem extensas áreas de cultivo de cana de açúcar e carcinocultura. A comunidade ribeirinha é constituída por cerca de 66.000 habitantes. A foz do rio forma uma baía de seis quilômetros de largura margeada por uma linha de recifes de arenito, o que resulta em águas calmas e tranquilas. O estuário exibe manguezais bem conservados compostos principalmente por Rhizophora mangle, Avicennia schaueriana, Avicennia germinans, Laguncularia racemosa e Conocarpus erectus que crescem em torno do canal principal e por riachos intertidais, compreendendo cerca de 6.000 hectares, há também remanescentes da Mata Atlântica no local.

Duas campanhas de amostragens foram realizadas no estuário, uma no pico da estação seca (novembro de 2013) e outra no pico da estação chuvosa (julho de 2014). Os peixes foram capturados via arrastos manuais, com rede de $10 \mathrm{~m}$ de comprimento X 1,5 m de altura e malha de $8 \mathrm{~mm}$. Os arrastos, com extensão de $30 \mathrm{~m}$, ocorreram em 12 pontos de amostragem, compreendendo as zonas do estuário (oligohalino, mesohalino, polihalino e euhalino) em período diurno na baixamar. No total foram realizados 72 arrastos no estuário do rio Mamanguape. Os espécimes coletadas foram fixadas em solução de formalina a $10 \%$ tamponada (Borato de Sódio), conduzidos ao laboratório e identificados com base em literatura pertinente. Em laboratório, foi medido o comprimento total $(\mathrm{mm})$ e o peso total $(\mathrm{g})$ dos indivíduos capturados. Para testar a correlação nos parâmetros morfométricos (peso total e comprimento total) das espécies coletadas no estuário, em cada zona e estação, foi utilizada uma análise multivariada baseada em permutação de variância (PERMANOVA, ANDERSON, 2001). Antes da análise, as variáveis foram normalizadas e os dados foram convertidos em uma matriz de similaridade utilizando a distância Euclidiana. Nós avaliamos a significância de $p \leq 0,05$, após 9999 permutações. 


\section{RESULTADOS E DISCUSSÃO}

A ictiofauna do estuário foi composta por 59 espécies, distribuídas em 11 ordens, 25 famílias e 40 gêneros, perfazendo 2.003 indivíduos capturados na seca e 1.635 na chuva. Perciformes foi a ordem mais representativa em termos de riqueza com 27 espécies e abundância de 434 indivíduos. As famílias mais representativas em número de espécies foram Engraulidae e Gobiidae, com 7 espécies cada, seguidas por Gerreidae, com 5 espécies. As demais famílias estiveram representadas por 4 ou menos espécies.

Dentre as espécies comuns nos períodos de seca e chuva, Atherinella brasiliensis, apresentou elevada abundância, com 699 e 375 indivíduos, respectivamente. Esta espécie é considerada residente estuarina e abundante em estuários tropicais (CONTENTE et al., 2010. Outras, embora não completem o ciclo de vida em estuários, foram comuns em algum dos períodos amostrados, é o caso de Mugil curema, 884 indivíduos na chuva, Anchoa januaria, 165 indivíduos, Lycenraulis grossidens, 103 indivíduos e Poecilia vivipara, 220 indivíduos, ambos no período seco. Estas espécies fazem uso do ambiente para proteção, crescimento ou reprodução (MARTINS e VENDEL, 2014) (Tabela 1).

Tabela 1. Médias do comprimento (mm, average \pm SD (min -max)) e peso ( g, average \pm SD (min max)) e Biomassa (g) das espécies de peixes ocorrentes no Estuário do Rio Mamanguape.

\begin{tabular}{|c|c|c|c|c|c|c|c|c|c|}
\hline \multirow[t]{2}{*}{ TAXA } & \multirow[t]{2}{*}{ SPECIES } & \multicolumn{4}{|c|}{ DRY } & \multicolumn{4}{|c|}{ WET } \\
\hline & & n & $\begin{array}{l}\text { Length }[\mathrm{mm} \text {, } \\
\text { average } \pm \text { s.d.] }\end{array}$ & $\begin{array}{l}\text { welght } \quad[\mathrm{g} \text {, } \\
\text { average } \pm \text { s.d.] }\end{array}$ & $\begin{array}{l}\text { Biomass } \\
{[\mathrm{g}]}\end{array}$ & n & $\begin{array}{l}\text { Length } \quad[\mathrm{mm} \text {, } \\
\text { average } \pm \text { s.d] }\end{array}$ & $\begin{array}{l}\text { weight [g, } \\
\text { average } \pm \text { s.d] }\end{array}$ & $\begin{array}{l}\text { Biomass } \\
{[\mathrm{g}]}\end{array}$ \\
\hline \multicolumn{10}{|l|}{ Anguilliformes } \\
\hline $\begin{array}{l}\text { Ophichthidae } \\
\text { Clupeiformes }\end{array}$ & Ophichthus cylindroideus (Ranzani, 1839) & 1 & 341 & 16.9 & 16,9 & $\cdot$ & - & - & \\
\hline \multirow[t]{4}{*}{ Clupeidae } & Harengula chupeola (Cuvier 1829) & 1 & 79 & 3.8 & 3.8 & . & . & - & . \\
\hline & Lile piquitinga (Schreiner \& Miranda Ribeiro, 1903) & $\cdot$ & - & $\cdot$ & . & 3 & $84.3 \pm 11.5$ & 5.6 & 16.9 \\
\hline & Opisthonema oglimum (Lesueur, 1818) & 80 & $147.4 \pm 9.8$ & $27.1+5.5$ & 2164.5 & - & - & - & $\cdot$ \\
\hline & Rhinosardinia bahiensis (Steindacluner, 1879) & 70 & $71.3 \pm 14.5$ & $2.7 \pm 1.4$ & 194.9 & 4 & $69.7 \pm 4.0$ & $2.3 \pm 1.9$ & 8.9 \\
\hline \multirow[t]{7}{*}{ Engraulidae } & Anchoa januaria (Steindachner, 1879) & 165 & $66.0 \pm 4.9$ & $2.0 \pm 0.4$ & 334.6 & 1 & 42 & 0.3 & 0.3 \\
\hline & Anchoa Iyolepis (Evermarn \& Marsh, 1900) & 1 & 75 & 2.78 & 2.7 & $\cdot$ & - & - & - \\
\hline & Anchoa marnini Hildebrand, 1943 & 7 & $54.7 \pm 12.6$ & $1.1 \pm 0.7$ & 7.8 & 5 & $49.2 \pm 12.9$ & $0.8 \pm 2.0$ & 4.3 \\
\hline & Anchoa spinifer Valenciernes, 1848 & - & - & - & - & 2 & $65.0 \pm 14.5$ & $1.6 \pm 1.0$ & 3.2 \\
\hline & Anchovia chupeoides (Swainson, 1839) & 9 & $114.5 \pm 39.8$ & $14.7 \pm 16.7$ & 132.5 & - & - & - & $\cdot$ \\
\hline & Anchovielia lepidentostde (Fowler, 1911) & 5 & $47.0 \pm 6.8$ & $0.6 \pm 0.32$ & 3.04 & 2 & $40.5 \pm 0.7$ & 0.3 & 0.7 \\
\hline & Lycengraulis grossidens (Spix \& Agassiz 1829) & 103 & $69.6 \pm 23.2$ & $3.3 \pm 6.8$ & 344.6 & 3 & $80.0 \pm 47.6$ & $6.3 \pm 9.6$ & 19.1 \\
\hline \multicolumn{10}{|l|}{ Characiformes } \\
\hline \multicolumn{9}{|l|}{ Siluriformes } & 3.8 \\
\hline $\begin{array}{l}\text { Ariidae } \\
\text { Batrachoidiforme }\end{array}$ & Sciades herzbergit (Bloch, 1794) & . & . & . & . & 3 & $56.3 \pm 2.8$ & $1.1 \pm 0.5$ & 3.5 \\
\hline $\begin{array}{l}\text { Batrachoididae } \\
\text { Atheriniformes }\end{array}$ & Thalassoplaryne nattereri Steindachner, 1876 & - & . & . & - & 1 & 132 & 49.9 & 49.9 \\
\hline
\end{tabular}




\begin{tabular}{|c|c|c|c|c|c|c|c|c|c|}
\hline \multirow[t]{2}{*}{ Atherinopsidae } & Atherinelia blackbtoni (Quoy \& Gaimard, 1825) & 12 & $55.0 \pm 3.0$ & $0.9 \pm 0.2$ & 11.3 & - & - & - & - \\
\hline & Atherinelia brasiliensis (Quoy \& Gaimard, 1825) & 699 & $52.6 \pm 22.5$ & $1.2 \pm 1.9$ & 905.5 & 375 & $79.4 \pm 25.0$ & $3.8 \pm 69.8$ & 1426.1 \\
\hline \multicolumn{10}{|c|}{ Cyprinodontiformes } \\
\hline Poeciliidae & Poectlia vivipara Bloch \& Schneider, 1801 & 220 & $38.5 \pm 9.4$ & $0.9 \pm 0.6$ & 201.9 & - & - & - & . \\
\hline \multicolumn{10}{|l|}{ Beloniformes } \\
\hline \multirow[t]{2}{*}{ Belonidae } & Strongylira marina (Walbaum, 1792) & 5 & $134.8=15.1$ & $3.2 \pm 1.8$ & 16.4 & - & - & . & . \\
\hline & Strongylara timucu (Walbaum, 1792) & 1 & 261 & 11,25 & 11,2 & 3 & $288.6 \pm 25.1$ & $27.5 \pm 22.5$ & 82.5 \\
\hline \multirow[t]{2}{*}{ Hemiramphidae } & Hyporhamphus roberti roberti (Valenciennes, 1847) & 2 & $145 \pm 21.2$ & $5.7 \pm 2.6$ & 11.4 & 2 & $150 \pm 16.9$ & 6.2 & 12.3 \\
\hline & Hyporhamphus tunifasciatus (Ranzani, 1841) & 91 & $124.8 \pm 28.2$ & $4.0 \pm 2.4$ & 367.0 & 34 & $151.6 \pm 21.0$ & $7.3 \pm 30.5$ & 249.6 \\
\hline \multicolumn{10}{|l|}{ Perciformes } \\
\hline Centropomidae & Centropormus undecimalis (Bloch, 1792) & 3 & $178.6 \pm 75.4$ & $46.0 \pm 47.2$ & 138.2 & 1 & 156 & 25.8 & 25.8 \\
\hline Serranidae & Rypticus randalli Courtenay, 1967 & 2 & $56.0 \pm 4.2$ & $1.6 \pm 0.2$ & 3.2 & - & - & - & . \\
\hline \multirow[t]{4}{*}{ Carangidae } & Caranx latus Agassiz, 1831 & 4 & $73.7 \pm 15.9$ & $5.6 \pm 3.9$ & 22.5 & 29 & $46.0 \pm 10.4$ & $4.4 \pm 25.8$ & 128.5 \\
\hline & Oligaplites palometa (Cuvicr, 1832) & 11 & $45.9 \pm 12.3$ & $0.9 \pm 0.6$ & 10.5 & - & $\cdot$ & $\cdot$ & $\cdot$ \\
\hline & Oligoplites saliens (Bloch 1793) & 1 & 78 & 3,2 & 3,2 & - & $\cdot$ & $\cdot$ & $\cdot$ \\
\hline & Oligoplites saurus (Bloch \& Schneider, 1801) & 5 & $68.2 \pm 13.3$ & $2.2 \pm 0.9$ & 11.0 & 4 & $43.0=12.7$ & $0.7 \pm 0.3$ & 2.8 \\
\hline \multirow[t]{2}{*}{ Lutjanidae } & Lutjanus alexandrei Moura \& Lindeman, 2007 & 2 & $32.5 \pm 0.7$ & $0.5 \pm 0.07$ & 1.1 & - & $\cdot$ & $\cdot$ & $\cdot$ \\
\hline & Lutjanus jocu (Bloch \& Schneider, 1801) & 2 & $165.0=66.4$ & $84.5 \pm 80.5$ & 169.0 & - & $\cdot$ & $\cdot$ & $\cdot$ \\
\hline \multirow[t]{5}{*}{ Gerreidae } & Diapterns rhombeus (Cuvier, 1829) & 22 & $55.5 \pm 20.2$ & $3.1 \pm 4.8$ & 68.3 & - & $\cdot$ & $\cdot$ & - \\
\hline & Diapterus aurratus Ranzani 1842 & 54 & $53.4 \pm 9.1$ & $1.7 \pm 1.0$ & 94.4 & - & $\cdot$ & $\cdot$ & $\cdot$ \\
\hline & Euctnostomus argenteus Baird \& Girard, 1855 & 81 & $49.4 \pm 12.9$ & $1.6 \pm 2.9$ & 133.5 & 19 & $39.4 \pm 15.5$ & $0.6 \pm 5.5$ & 11.7 \\
\hline & Euctnostomis melanoptenus (Bleeker, 1863) & 3 & $50.3 \pm 1.5$ & $1.2 \pm 0.1$ & 3.6 & 25 & $38.5 \pm 11.0$ & $0.3 \pm 1.4$ & 8.3 \\
\hline & Eugerres brastitianus (Cuvier, 1830) & 134 & $51.5 \pm 10.3$ & $1.7 \pm 1.7$ & 231.7 & 27 & $52.5 \pm 8.0$ & $1.6 \pm 11.6$ & 43.1 \\
\hline Haemulidae & Pomadasys corvinaeformis (Steindachner, 1868) & 3 & $125.6 \pm 13.2$ & $26.0 \pm 7.7$ & 78.2 & 1 & 119 & 23.0 & 23.0 \\
\hline Sciaenidae & Steilifer stellifer (Bloch, 1790) & 1 & 142 & 27,65 & 27,65 & - & $\cdot$ & $\cdot$ & - \\
\hline \multirow[t]{3}{*}{ Mugilidae } & Mugil curema Valenciennes, 1836 & 9 & $97.8 \pm 44.1$ & $15.9 \pm 15.7$ & 143.9 & 884 & $27.6 \pm 4.7$ & $0.2 \pm 29.5$ & 194.3 \\
\hline & Mugil curvidens Valenciennes 1836 & 66 & $47.8 \pm 16.6$ & $2.0 \pm 6.7$ & 135.1 & - & $\cdot$ & $\cdot$ & $\cdot$ \\
\hline & Mugil liza Valenciennes, 1836 & 1 & 35 & 0,49 & 0,49 & 6 & $26.1=2.9$ & 0.1 & 1.0 \\
\hline Cichlidae & Oreochromis niloticus (Linnaeus, 1758) & 6 & $147.8 \pm 13.8$ & $60.4 \pm 19.8$ & 362.2 & - & - & - & - \\
\hline Eleotridae & Erotelis smaragdus (Valenciennes, 1837) & 1 & 32 & 0,19 & 0,19 & 1 & 67 & 1.5 & 1.5 \\
\hline \multirow[t]{7}{*}{ Gobiidae } & Bathygobius soporator (Valenciennes, 1837) & 5 & $76.8 \pm 13.4$ & $6.7 \pm 3.6$ & 33.9 & 5 & $76.8 \pm 19.5$ & $6.5 \pm 3.3$ & 33.7 \\
\hline & Ctenogobtus boleosoma (Jordan \& Gilbert, 1882) & 13 & $36.0 \pm 5.3$ & $0.3 \pm 0.1$ & 4.6 & 21 & $28.2 \pm 9.8$ & $0.1 \pm 0.2$ & 3.7 \\
\hline & Ctenogobtus sinffeldti (Jordan \& Eigenmann, 1887) & 1 & 45 & 0.57 & 0,57 & - & $\cdot$ & $\cdot$ & $\cdot$ \\
\hline & Chenogobfus smaragdus (Valenciennes, 1837) & 1 & 21 & 0.05 & 0,05 & - & $\cdot$ & $\cdot$ & $\cdot$ \\
\hline & Gobionellus aceanicus (Pallas, 1770) & $\cdot$ & - & - & & 4 & $181.0=40.4$ & $25.4 \pm 33.0$ & 101.8 \\
\hline & Gobionellus stomatus Starks 1913 & 1 & 60 & 0,81 & 0,81 & 8 & $49 \pm 16.6$ & $0.7 \pm 1.6$ & 5.6 \\
\hline & Microgobius meeki Evermarn \& Marsh, 1899 & 2 & $38.0 \pm 1.4$ & $0.2 \pm 0.01$ & 0.5 & - & $\cdot$ & - & $\cdot$ \\
\hline \multicolumn{10}{|l|}{ Pleuronectiformes } \\
\hline \multirow[t]{3}{*}{ Paralichthyidae } & Citharichthys macrops Dresel, 1885 & 15 & $52.0 \pm 15.2$ & $1.3 \pm 1.1$ & 20.0 & 5 & $52.6 \pm 44.3$ & $4.4 \pm 10.2$ & 22.3 \\
\hline & Citharichthys spilcpterns Gūnther, 1862 & 9 & $111.1 \pm 27.7$ & $14.4 \pm 9.3$ & 129.7 & - & $\cdot$ & $\cdot$ & \\
\hline & Etropus crossotus Jordan \& Gilbert, 1882 & 1 & 30 & 69.2 & 69.2 & - & - & - & . \\
\hline \multirow[t]{2}{*}{ Achiridae } & Achirus dectivis Chabanaud, 1940 & 11 & $62.9 \pm 36.4$ & $9.8 \pm 17.0$ & 108.8 & - & $\cdot$ & $\cdot$ & $\cdot$ \\
\hline & Achinus lineatus (Linnaeus, 1758) & 17 & $44.6 \pm 12.7$ & $1.8 \pm 1.6$ & 31.7 & 3 & $70.0 \pm 12.5$ & $6.0 \pm 3.9$ & 18.1 \\
\hline Cynoglossidae & Symphurus tessellatus (Quoy \& Gaimard, 1824) & 5 & $71.2 \pm 9.6$ & $2.0 \pm 0.9$ & 10.3 & 2 & $150.5 \pm 3.5$ & $21.3 \pm 1.6$ & 42.6 \\
\hline \multicolumn{10}{|l|}{ Tetraodontiformes } \\
\hline \multirow[t]{4}{*}{ Tetraodontidae } & Colomesus psittacus (Bloch \& Schneider, 1801) & $\cdot$ & - & - & - & 6 & $108.3 \pm 10.7$ & 28.1 & 169.1 \\
\hline & Lagocephalus laevigatus (Linnaeus, 1766) & 1 & 198 & 131.2 & 131.2 & - & $\cdot$ & $\cdot$ & $\cdot$ \\
\hline & Sphoeroides greeieyi Gilbert, 1900 & 4 & $18.7 \pm 2.5$ & $0.1 \pm 0.04$ & 0.4 & 16 & $71.1 \pm 7.5$ & $7.5 \pm 16.9$ & 121.1 \\
\hline & Sphoeroides testudineus (Linnaeus, 1758) & 31 & $31.8 \pm 20.2$ & $1.7 \pm 4.9$ & 53.8 & 129 & $64.9 \pm 33.7$ & $7.5 \pm 107.8$ & 974.9 \\
\hline
\end{tabular}

No estudo, nós avaliamos a correlação PT-CT interespecífica por estação e foi possível observar altos coeficientes de correlação das relações peso-comprimento para a grande parte das espécies na seca $\left(r^{2}=0,9684\right)$ e na chuva $\left(r^{2}=0,9997\right)$. Observamos ainda, a significância intraespecífica das espécies e, de acordo com a análise, o valor de "p" para a maioria das espécies não foi significativo, com exceção de $L$. jocu $(0,0078)$ na seca, e E. brasilianus $(\mathrm{p}=0,0007)$ e $H$. unifasciatus $(0,0039)$ na chuva. Os indivíduos destas espécies apresentaram elevados valores de peso e comprimento, quando comparados às outras espécies da amostra e, possivelmente, isto pode ter influenciado na correlação significativa entre peso e comprimento destes indivíduos. 

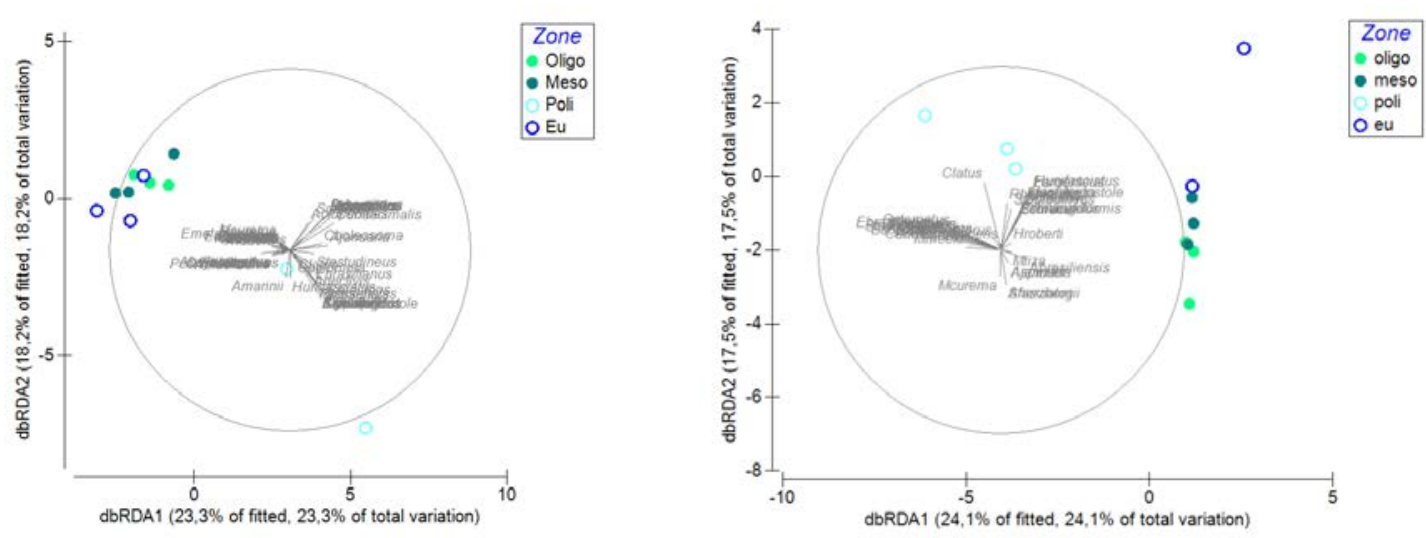

Figura 2. Análise de redundância (RDA) entre peso e comprimento da ictiofauna do Estuário do Rio Mamanguape.

Por fim, foi realizada a análise de redundância entre os dados de peso e comprimento para todos os indivíduos da amostra. Tal relação foi testada em separado para a estação seca (Figura 2a) e chuvosa (Figura 2b) e segundo a zona do estuário. Na estação seca, a variação não colinear explicou $41,5 \%$ da variação total, enquanto na chuva, explicou $41,6 \%$ da variação total. A análise destacou o setor polihalino devido às altas capturas de indivíduos nesta zona, com peixes de maior porte na estação seca, em oposição às baixas capturas de indivíduos menores nas demais zonas do estuário, em ambas as estações.

\section{CONCLUSÕES}

O estudo representa uma importante contribuição ao conhecimento biológico da ictiofauna local. Os resultados obtidos destacam a importância de áreas estuarinas como habitats para peixes residentes estuarinos e visitantes marinhos e de água doce. Além de contribuir para o conhecimento das relações de CT-PT das populações de teleósteos ocorrentes neste estuário, proporciona dados que contribuem na gestão da comunidade de peixes.

\section{REFERÊNCIAS}

ANDERSON, M. J. Permutation tests for univariate or multivariate analysis of variance and regression. Canadian Journal of Fisheries and Aquatic Sciences, v. 58, p. 626-639, 2001.

ARAÚJO, M. E.; TEIXEIRA, J. M. C.; OLIVEIRA, A. M. E. Peixes Estuarinos do Nordeste Brasileiro: Guia Ilustrado. Fortaleza: Edições UFC, 2004.

BLABER, S. J. M. Tropical estuarine fishes: ecology, exploitation and conservation. Oxford: Blackwell Science, 2000.

CONTENTE, R. F., STEFANONI, M. F. \& SPACH, H. L. Feeding ecology of the Brazilian silverside Atherinella brasiliensis (Atherinopsidae) in a sub-tropical estuarine ecosystem. Journal of the Marine Biological Association of the United Kingdom, v. 91, 2010.

CORRÊA, F.; OLIVEIRA, E. F.; POUEY, J.; PIEDRAS, S. Length-weight relationship of trahira Hoplias aff. Malabaricus (Bloch, 1794) in a regulated stream of southern Brazil. Biotemas, v. 29, n. 2, p. 169-174, 2016. 
GODEFROID, R. S.; SPACH, H. L.; SCHWARZ Jr, R.; QUEIROZ, G. M. L. N.; OLIVEIRA NETO, J. F. Efeito da lua e da maré na captura de peixes em uma planície de maré da Baía de Paranaguá, Paraná, Brasil. B. Inst. Pesca, São Paulo, v. 29, n. 1, p. 47-55, 2003.

KENNISH, M. J. Ecology of Estuaries. Biological Aspects. Florida: CRC Press, 1990.

LIZAMA, M. A.; AMBRÓSIO, A. M. Crescimento, recrutamento e mortalidade do pequi Moenkhausia intermédia (Osteichthyes, Characidae) na planície de inundação do Alto Rio Paraná, Brasil. Acta Scientiarum, v. 25, n. 2, p. 329-333, 2003.

MARTINS, T. O.; VENDEL, A. L. Fishes collected with an artisanal fish trap in Barra de Camaratuba Estuary, Northeastern Brazil.Check List, v. 10, 1260, 2014.

VIANNA, M., COSTA, F. E. S.; FERREIRA, C. N. Length-weight relationship of fish caught as by-catch by shrimp fishery in the Southeastern coast of Brazil. Bol. Inst. Pesca, v. 30, n. 1, p. 81-85, 2004. 